\title{
MEASUREMENT OF $\eta$ IN THE ACCUMULATOR
}

\author{
M. Church
}

20 February 1992

\section{Introduction}

E760 uses the longitudinal Schottky spectrum of the circulating beam in the Accumulator to extract the momentum spectrum:

$$
\Delta p / p=\frac{1}{\eta} * \Delta f / f
$$

Hence it is required to accurately know $\eta$ at all energies. $\eta$ can be measured to about $5 \%$ by measuring the synchrotron frequency of bunched beam vs. the RF voltage and using the relation

$$
\eta=\frac{2 \pi f_{s}^{2} \beta^{2} E}{H f_{0}^{2} e \hat{V}}
$$

ARF3 ( $\mathrm{H}=2)$ was used in this case.

\section{Calculation}

The particle motion in longitudinal phase space, in a stationary RF bucket is given by the equations:

$$
\frac{d(\delta E)}{d t}=e \hat{V} f_{0}\left[g(\phi)-g\left(\phi_{s}\right)\right], \quad \frac{d(\delta \phi)}{d t}=\frac{-2 \pi H f_{0} \eta}{\beta_{2} E} \delta E,
$$

where $\delta E$ is the difference from the synchronous energy, $\delta \phi$ is the difference from the synchronous phase, $\phi_{s}$ is the synchronous phase, $H$ is the harmonic number, $f_{0}$ is the revolution frequency, $e \hat{V}$ is the maximum RF voltage, and

$$
g(\phi)=\sum_{n=1} A_{n} \sin \left(n \phi+\delta_{n}\right)
$$

The 2nd order differential equation for $\delta \phi$ is

$$
\frac{d^{2}(\delta \phi)}{d t^{2}}=\frac{-2 \pi H f_{0}^{2} \eta e \hat{V}}{\beta^{2} E}\left[g(\phi)-g\left(\phi_{s}\right)\right]
$$


Choosing $A_{1}=1$ and $\delta_{1}=0$ and using the small angle approximation we get

$$
g(\phi)-g\left(\phi_{\bullet}\right)=\delta \phi\left[\cos \left(\phi_{\bullet}\right)+\sum_{n=2} n A_{n} \cos \left(n \phi_{\iota}+\delta_{n}\right)\right]
$$

and

$$
f_{s}=\left\{\frac{H f_{0}^{2} \eta e \hat{V}}{2 \pi \beta^{2} E}\left[\cos \left(\phi_{s}\right) \sum_{n=2} n A_{n} \cos \left(n \phi_{s}+\delta_{n}\right)\right]\right\}^{\frac{1}{2}}
$$

If the higher harmonics of the RF are not 0 , then $\phi_{s}$ is not necessarily 0 . The energy imparted per turn to a particle from the fundamental RF mode is

$$
\Delta E_{1}=e \hat{V} \sin \left(\phi_{s}\right)
$$

and from the higher modes is

$$
\Delta E_{2,3 \ldots}=\sum_{n=2} A_{n} \sin \left(n \phi_{s}+\delta_{n}\right)
$$

For the synchronous particle $\Delta E_{1}=-\Delta E_{2,3 \ldots}$ and assuming $\phi_{0}$ is small we get

$$
\phi_{s}=\frac{-\sum_{n=2} A_{n} \sin \left(\delta_{n}\right)}{1+\sum_{n=2} n A_{n} \cos \left(\delta_{n}\right)} .
$$

\section{Measurements}

The power in the higher harmonics in ARF3 was measured with a spectrum analyzer. They are $50.4 \mathrm{dBm}, 63.5 \mathrm{dBm}, 78.6 \mathrm{dBm}, 74.2 \mathrm{dBm}$, and $79.4 \mathrm{dBm}$ down from the fundamental harmonic at $\mathrm{n}=2,3,4,5,6$ respectively. These were measured at the patch panel in AP10 and may contain nonlinear gain effects in amplification between AP50 and AP10 - hence they represent an upper limit. Although the phases, $\delta_{n}$, were not measured, the worst case can be seen to be (from eq. 10): $\delta_{n}=0$ for even harmonics and $\delta_{n}=\pi$ for odd harmonics above transition; and $\delta_{n}=0$ for all harmonics below transition. A numerical calculation with these values gives the magnitude of the correction term in eq. 7 of less than $.5 \%$. Therefore, the effects of higher harmonics can be neglected, and eq. 2 is valid in this case.

The RF voltage was measured directly across the gap using scope probes and this was used to calibrate the fanback signals at both AP10 and AP50. They are $18.4 \mathrm{kV} / \mathrm{V}$ at $\mathrm{AP} 10$ and $2.538 \mathrm{kV} / \mathrm{V}$ at AP50. The uncertainty in this calibration is about $5 \%$, and this is the major uncertainty in the measurement of $\eta$.

The synchrotron frequency was measured by mixing the signal from the gap monitor or the longitudinal Schottky pickup down to $10 \mathrm{kHz}$ with a signal from an $\mathrm{HP}$ 3335A signal generator using a ZAD-1-1 linear mixer. This signal was then frequency analyzed with an HP 3562A dynamic signal analyzer. A typical signal from the gap monitor is shown in Fig. 1 and a typical signal from the longitudinal Schottky pickup is shown in Fig. 2 showing the synchrotron sidebands. The synchrotron frequency is taken from the outer edge of the sidebands, as that corresponds to small amplitude synchrotron motion.

The RF voltage was measured by measuring the rms power in an HP $436 \mathrm{~A}$ power meter at AP50 or the rms power in an HP $8568 \mathrm{~B}$ spectrum analyzer at AP10. 
Eq. 2 can be rewritten in more convenient form as

$$
\eta=\frac{9.9354 \times 10^{-6}\left(P_{b} c\right) f_{s}^{2}}{F_{r f}(e \hat{V})}
$$

where I have used an orbit length of $L=474.046$ meters, and the units are $\mathrm{Hz}$ for $f_{s}$, Gev/c for $P_{b} c, \mathrm{kV}$ for $e \hat{V}$, and Mhz for $F_{r f}$. A plot of $f_{a}^{2}$ vs. $\sqrt{P_{r m s}}$ should yield a straight line whose slope is proportional to $\eta$. If $P_{r m s}$ is measured in $\mathrm{mW}$, then

$$
\eta=\text { slope } \times C \times P_{b} c / F_{r f}
$$

where $C=.391 \times 10^{-6}$ for power measured at AP50 and $C=.0540 \times 10^{-6}$ for power measured at AP10. A typical plot of $f_{a}^{2}$ vs. $\sqrt{P_{\text {rms }}}$ is shown in Fig. 3. 


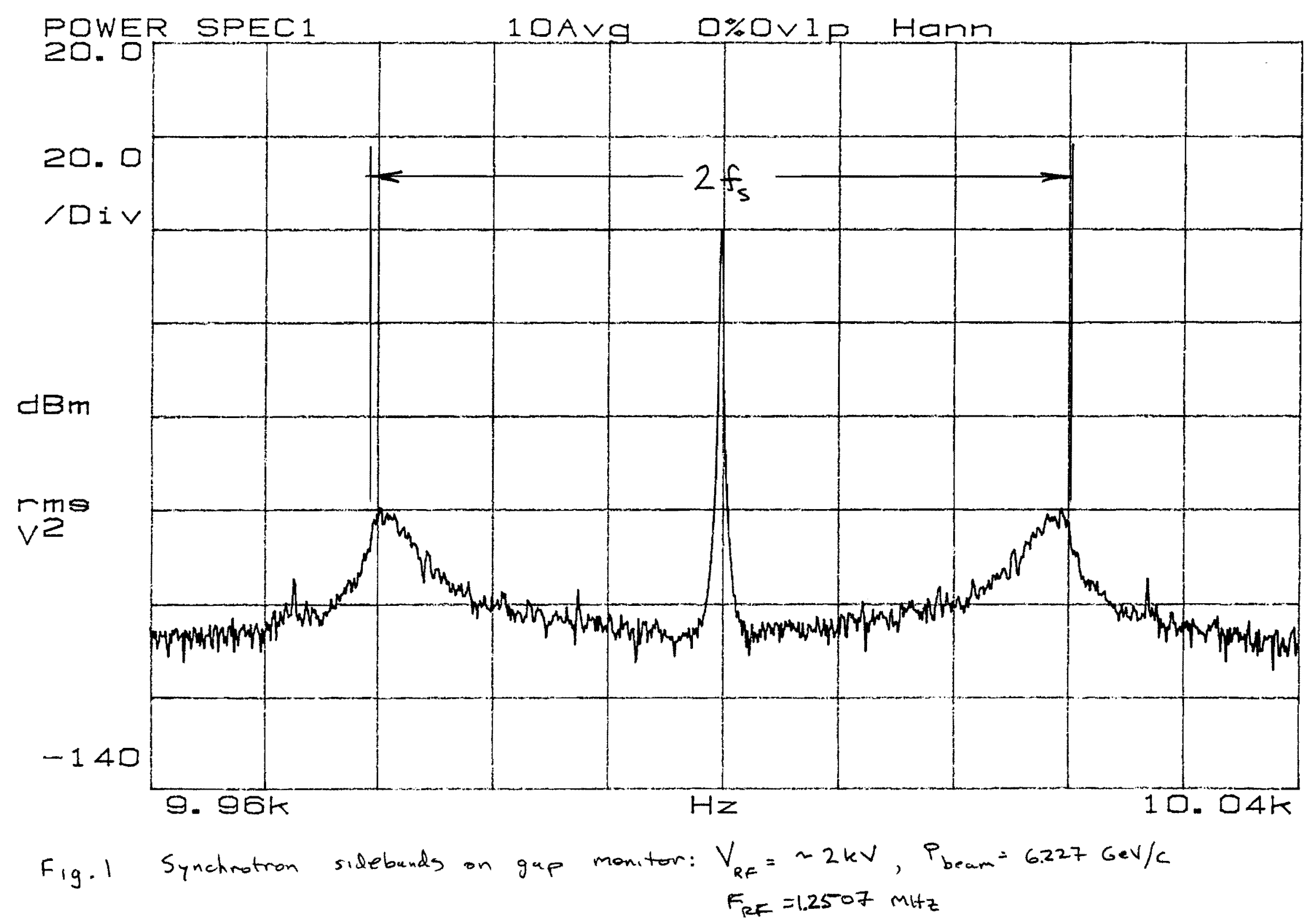




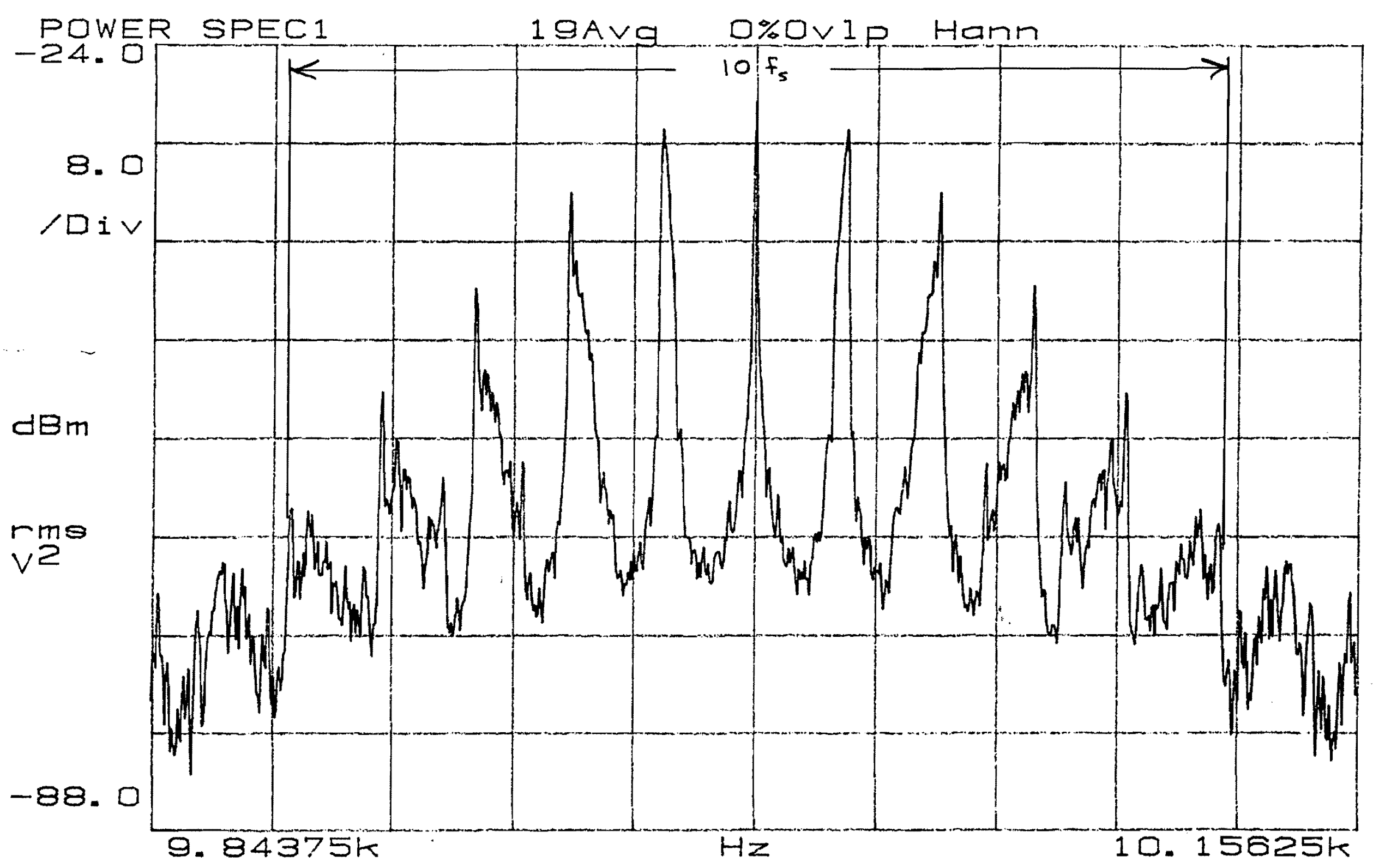

Fig. 2 Synchrotion sidebunds on schottly piateop. $V_{R F} \sim 2 \mathrm{kV}, P_{\text {beam }}=6.227 \mathrm{ceV} / \mathrm{C}$
$F_{R F}=1.2507 \mathrm{MHz}$ 


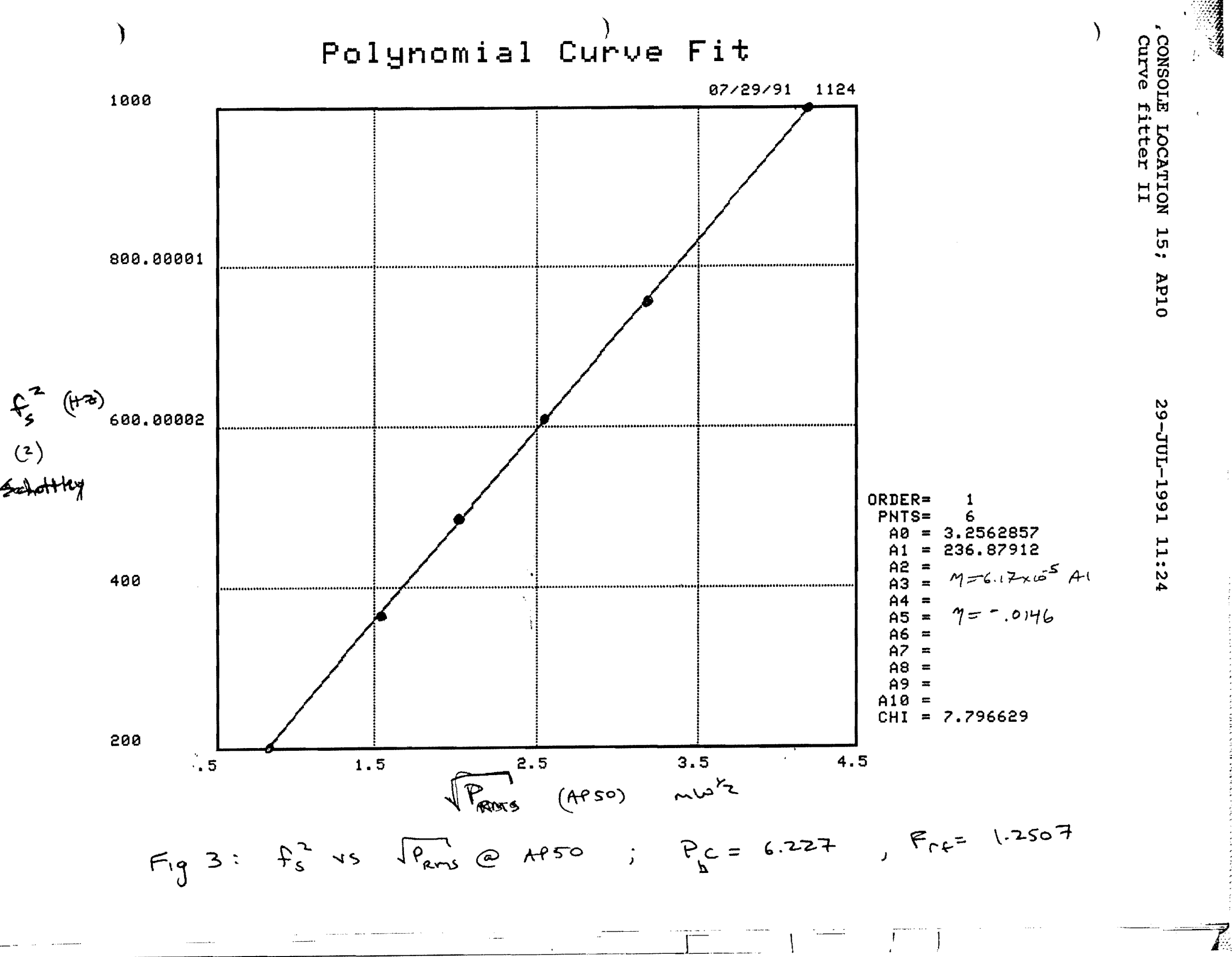

